\title{
HEALTH EFFECTS OF SHIFT WORK AND EXTENDED HOURS OF WORK \\ J M Harrington
}

\section{INTRODUCTION}

"Normal" hours of work are generally taken to mean a working day with hours left for recreation and rest. Rest is a night time activity, work a daytime activity. This review is concerned with those who work other schedules either on shifts or with extended hours which transcend the day-night work-sleep pattern.

Such "abnormal" working hours are not a modern phenomenon. Ramazzini (1633-1714) noted that bakers, innkeepers, and soldiers worked such hours. The advent of the industrial revolution led to many people working long hours until legislation was introduced to curtail the worst vicissitudes of the new factory based economy.

\section{Shift schedules}

Today, about one in five workers in Europe are employed on shift work involving night work and over one in 20 work extended hours . Shift systems involve periods of 6-12 hours work at a time with the shift crews alternating on two, three, or four shifts in any 24 hour period. The traditional three shifts start at 0600, 1400, and 2200 hours but there are many variations on this. Some workers only work on the two day shifts, some only nights, while others rotate through all three shifts with variable degrees of speed of rotation and direction of rotation. Extended hours of work is generally accepted to mean working more than 48 hours a week. This can occur on either day work or shift work due to either a high number of hours worked per day or a higher number of days worked per week.

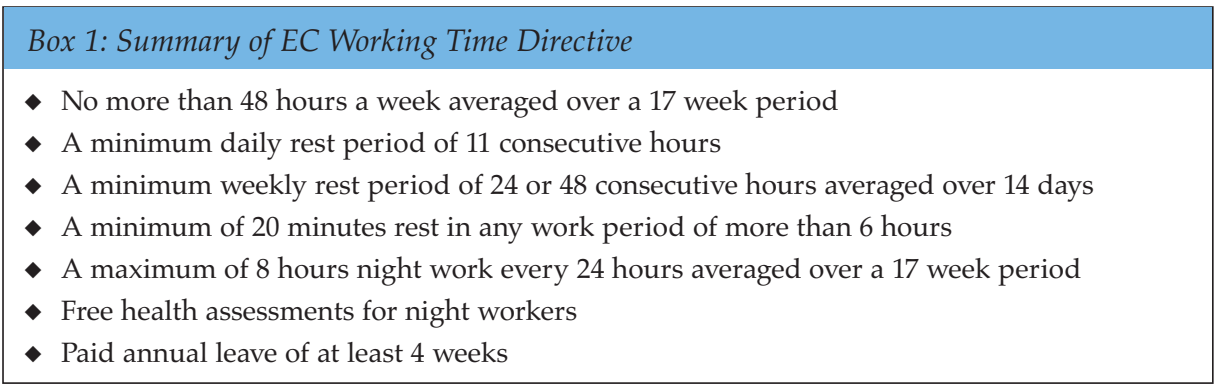

\section{Working time directive}

During the 1990s, substantial changes took place in international legislation on working time. The new European Directive on Working Hours (1993) introduced specific measures relating to the scheduling of shifts and rest periods whereas on a broader scale, the International Labour Organisation (ILO) introduced in 1990 radical new standards for working patterns. The object of each of these measures was to limit hours worked because long or abnormal work patterns are deemed to be hazardous to health. The main features of the European Directive as incorporated into United Kingdom law are found

Correspondence to: Professor J M Harrington Institute of Occupational Health, University of Birmingham, Birmingham B15 2TT, UK j.m.harrington@bham.ac.uk in box 1 . The reasons why shift work and now extended work has continued into the 21st century are threefold.

- Society's needs for services and emergency cover

- Technical need for maintaining continuous process industries

- Economic need for offsetting plant obsolescence. 
Although the shift worker of 50 years ago was likely to be factory based, increasing demand for services, both business and pleasure, has extended to those employed in more traditionally "white collar" occupations. E-commerce will lead to the need for 24 hour services for most retail and service organisations.

There are extensive publications on the health and social effects of shift work.

The quality of the papers does not always match the quantity. There are considerable methodological issues concerned with this subject. The most obvious is the fact that a large proportion of shift workers are a self selected population and those that remain shift workers for years are a survivor population. Many of the studies published are cross sectional, as there are difficulties in selecting appropriate comparison populations for longitudinal studies. Finally, some of the outcome measures in studies of shift work involve relatively "soft" measures such as fatigue, mood, and performance. Nevertheless, some excellent studies have provided the basis for what follows in this article. Some recent reviews have also highlighted the current state of knowledge in this area. ${ }^{1-4}$

\section{BIOLOGICAL AND SOCIAL ASPECTS}

\section{Circadian rhythms}

One of the most important physiological problems associated with shift work and the night shift in particular, is that working, eating, and sleeping phases are changed. Mammals have a natural rhythmicity to many bodily functions and these circadian rhythms exist in humans, with many operating on a 25 hour cycle. Such free running cycles, which include body temperature, respiratory rate, urinary excretion, cell division, and hormone production, can be modulated by exogenous factors such as light-dark cycle, social climate, and of course, work schedules.

For example, under normal living conditions, body temperature peaks in the late afternoon with its lowest point occurring in the early hours of the morning. Under experimental conditions, it is possible to reverse this cycle but rotating shift workers usually only succeed in flattening the curves. Body temperature records are often used as a surrogate measure of disruption of circadian rhythm, but it is probably too simplistic to link this measure directly to performance. ${ }^{5}$

\section{Efficiency of performance}

Human error is often cited as an important factor in work accidents and this may depend to some extent on sleep related factors and circadian rhythm. In general, efficiency of performance seems to parallel the circadian variation in body temperature. The disruption of circadian rhythm, combined with sleep deficit and fatigue, can lead to workplace inefficiency, particularly in the early hours of the morning. This pattern of cause and effect has been reported for many groups of shift workers. A dip after lunch has also been described for lowered efficiency of performance and this is only partially dependent on the meal itself. ${ }^{6}$
The shortening of the sleep period caused by an early start at work has also been shown to be associated with an increase in errors and accidents in transport workers.

\section{Family and social life}

Workers who engage in shift work or who work long hours can experience considerable disruption of family and social activities as many of these rhythms of the general population are oriented around the day. Saturday and Sunday work, for example, can preclude involvement in sporting events or religious activities. Shift work can thus lead to social marginalisation.

Family and marital responsibilities can be severely disrupted by shift work or long hours. Childcare, housework, shopping, and leaving a partner alone at night can all lead to marital strain and family dysfunction. On the positive side, for those shift workers who like relatively solitary leisure pursuits or who abhor the crowds often find that shift scheduling provides them with greater opportunities to do what they want to do in their nonworking time.

\section{HEALTH EFFECTS}

Box 2: Summary of health effects of shift work
- Reduction in quality and quantity of sleep
- Widespread complaints of "fatigue"
- Inxiety, depression, and increased neuroticism
- Possible increase in gastrointestinal disorders
- Increased risk of spontaneous abortion, low birth weight,
and prematurity

\section{Sleep}

There is general agreement in publications that the effects of long hours of work or shift work has a deleterious effect on sleep. Perhaps the most authoritative review concludes that despite considerable variation between people, sleep loss is a major effect of shift work. ${ }^{7}$ This is most noticeable after the night shift. The quantity of sleep may be reduced by up to 2 hours a day but there is also an effect on the quality of sleep. Rapid eye movement (REM) sleep and stage 2 sleep have been shown to be reduced. Such sleep deficits can lead to sleepiness at work, with some data showing that inadvertent napping at work can result. Overall, the effect of shift work has been likened to a long distance traveller working in San Francisco and returning to London for any rest days.

\section{Fatigue}

Fatigue is a common complaint among those working abnormal hours. It is particularly noticeable after the night shift, less so on the morning shift, and least on the afternoon shift. Fatigue, however, is a complaint that is exceedingly difficult to measure. Some published 
evidence exists to suggest that there is a reduction in complaints of fatigue after objective improvement in physical fitness. Nevertheless, it remains an important, if vague, symptom which is often cited as a major reason for intolerance to shift work.

\section{Mental health}

Shift working can be a potential psychosocial stressor. Stress is, however, a difficult concept to define, let alone measure. Many published reports on working hours cite stress as a problem but such reports all too commonly lack scientific rigour, acquiring these data from self administered questionnaires and case reports. The increasing use of validated questionnaires such as the general health questionnaire $(\mathrm{GHQ})$ and, more specifically, the standard shift work index (SSI) developed by Barton and Folkard8 has improved the researchers' ability to characterise and measure the effects of shift work on mental health.

Anxiety and depression indices also point to the likelihood of an adverse effect on mental health from shift work and long working hours. Nevertheless it must be remembered that, by and large, shift workers are a self selected population. Thus the question of whether shift work causes psychiatric morbidity or shift workers have pre-existent psychiatric problems is not entirely resolved. There seems to be increased neuroticism with increasing years of shift work, but neuroticism in itself does not predict health related shift problems.

\section{Cardiovascular disorders}

In 1978, the general consensus was that there was no firm evidence that cardiovascular disease was more prevalent in shift workers than other groups. ${ }^{9}$ Today, that opinion would have to be revised, although much of the new evidence comes from Scandinavian studies. A recent review of the data suggests that shift workers have a $40 \%$ increase in risk. ${ }^{10}$ Causal mechanisms are not well defined but contributing factors include disruption of circadian rhythm, disturbed sociotemporal patterns and social support, stress, smoking, poor diet, and lack of exercise. The health outcomes are mainly angina pectoris, hypertension, and myocardial infarction.

The effect of overtime or long hours of work has been less extensively investigated. One mortality study from California showed increased rates of arteriosclerotic heart disease for male occupational groups in increasing proportions of the population who worked more than 48 hours a week. ${ }^{11}$ The 48 hour week cut off was an arbitrary one with information taken from censuses, and the study has not been replicated. The publicity surrounding the Japanese phenomenon of Karoshi (sudden vascular death) and overwork is relevant here but the published studies are little more than case series and thus lack epidemiological rigor.

\section{Gastrointestinal disorders}

Many shift workers complain of digestive disorders, which may be a reflection on the poor quality of catering on some shifts. Night workers seem to have the most complaints of dyspepsia, heartburn, abdominal pains, and flatulence. The data in these studies are not particularly robust. It is necessary to realise that psychosomatic disorders are common in the general population and to recognise the influence of several other factors including Helicobactor pylori, infection, family history, and lifestyle.

\section{Reproductive effects}

There is increasing evidence to suggest that shift work and particularly night work, may present special risks to women of child bearing age. ${ }^{4}$ The causative factors probably include disruption of the menstrual cycle and increased stress from the conflicts created by night work on family life. Specific health outcomes linked to shift work include increased risk of spontaneous abortion, low birth weight, and prematurity. The evidence for subfertility is less convincing.

\section{SAFETY EFFECTS}

\section{Box 3: Summary of safety effects of shift work}

- Poorer performance especially at night

- Increased rate of accidents especially at night

\section{Performance}

This area has already been alluded to. Until relatively recently, much of the evidence for a performance decrement associated with long hours of work relates to some excellent studies undertaken by Vernon et all1 on munitions workers in the first world war. The studies are thorough, well designed, and clearly show that reducing hours of work by between 7 and 20 per week (down to 50-55 hours per week) resulted in an improvement in the quality and quantity of units produced. Vernon et al also showed that pauses of 10 minutes in the morning and afternoon could increase production by $5 \%-12 \%$.

Since these studies, much of the research effort has been concentrated on laboratory based work because comparability of working conditions between shifts is difficult to find in the work situation. Some well designed workplace studies, however, support the laboratory based research. In short, there is a persuasive body of evidence to suggest that performance (output) is poorer at night. However, performance of tasks with a high working memory component is less dependent on endogenous circadian rhythm and thus may have relatively rapid adjustment to night work. This implies the need to view the task as well as the person in tailoring the shift system.

\section{Accidents}

The cause of industrial accidents is exceedingly complex but the link between increased fatigue with lowered performance and subsequent high rates of accidents would seem logical. Publications on working hours do not support this very well, largely because many of the stud- 
ies have inadequate analytical procedures, or failed to allow for the many confounding factors that can influence accident statistics. Furthermore, the night shift, which might intuitively be associated with more accidents, is often the time for "easy runs" of a particular task on what is often a less supervised shift. However, some recent well designed studies have shown an increased risk of accidents on the night shift and with long working hours. ${ }^{4}$

Several studies have shown peak accident rates at around 1000 and 1100 and again between 1300 and 1600 but this probably reflects peaks in work activities as performance capabilities due to circadian rhythms should be relatively high at these times.

Finally, on an anecdotal note, it is interesting that some major catastrophes such as Three Mile Island, Chernobyl, Exxon Valdez, and the space shuttle Challenger all started in the early hours of the morning with errors by people who had been on duty for long hours.

\section{Exposure to toxic chemicals}

This is an area that has received scant attention but clearly could be important in terms of long working hours or possible chemical variation in pharmokinetic handling of absorbed chemicals. What is clear is that occupational exposure limits are almost invariably calculated on the basis of an 8 hour day, 5 day week. Scaling down these limits to accommodate long working shifts such as 10 or 12 hours or making allowances for diurnal variation in metabolic processes is no easy task and no rules are available to accomodate these variations in working hours. There is a potential toxicological problem here but no solutions at present.

\section{PERSONAL ISSUES}

Box 4: Summary of personal issues and prevention
- Sex women>men
- Age older>younger
- Personality introversion/extroversion
- Physical fitness

\section{Sex}

Many countries have long banned the employment of women on shift work. The emergence of equal opportunity legislation has questioned the basis for such prohibition. It seems that women complain of more sleepiness on shift work (4) but the physiological basis for this is difficult to substantiate. Some of the increased difficulties that women have on shift work are related to their greater domestic obligations and to the broad based assertion that women tend to report health symptoms more often than men. Also there does seem to be some evidence for an adverse reproductive effect already noted.
Age

Intuitively, it might be proposed that shift working would be better tolerated the older the worker becomes. They might have less domestic pressures from small children, more experience of coping in general, and older people seem to require less sleep than younger people. Furthermore, the shift worker who has been working such schedules for decades is, of course, part of a survivor population.

In fact, the research findings support the opposite. The evidence all points in the direction of asserting that the aging worker tolerates shift work less well than younger colleagues. With age, sleep becomes shorter and more fragmented. This does not necessarily reflect a need for less sleep but simply an inability to achieve so easily what is needed. What is clear, therefore, is that whereas the aging shift worker may cope, it is inadvisable to begin shift work at an older age.

\section{Personality}

It has been proposed that almost one in five workers leave shift work because they cannot tolerate it, about $10 \%$ positively enjoy it, and the rest tolerate it to a greater and lesser extent. ${ }^{9}$ Personality may play a part in this as there is an association between neurotic introversion and intolerance to shift work. It may be simplistic to view introvert-extrovert personality types in this way but there is some physiological support for the importance of personality if people are viewed as morning types and evening types. People who are at their best in the morning face more difficulties in adjusting their circadian rhythms to night work compared with those who feel better in the evening. By contrast, evening types have greater problems with the early morning shift. Physical factors may also play a part and are discussed later.

\section{PREVENTION}

\section{Design of work schedule}

Most of the work on minimising the deleterious effects of shift work has concentrated on the three 8 hour shift rotation. As the night shift seems to cause a disproportionate amount of the problems of shift work, night work should be reduced as much as possible, perhaps by increasing the number of crews involved. Rapid rotation of shifts (a change every few days) is preferable to slow rotation as this schedule produces less interference with circadian rhythm. Clockwise rotation (morning, afternoon, night) is preferable to counter clockwise rotation as quick changeovers - for example, the morning and the night shift in the same 24 hour period-are avoided and this rotation allows longer rest periods between shifts. Finally, a later start for the morning shift which is traditionally 0600 reduces the truncation of the previous sleep period, particularly for REM sleep.

On balance, the duration of shift should not be extended to 10 or 12 hours as complaints of fatigue are greater

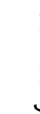


on the long shifts. However, the evidence that this translates into poorer performance and increased accidents is lacking. ${ }^{13}$ Many workers favour the longer shifts, not because they are longer but because the breaks between shift weeks are longer often 3-4 days.

Finally, there is some evidence that allowing the workers to design their own shift schedules encourages good work performance as they take responsibility for achieving the work output targets.

Other factors which can ameliorate shift schedules include workplace improvements in catering, supervision, health care, transportation, and recreational facilities. There is some limited evidence that bright light might be useful on the night shift to offset some of the circadian effects of the day-night changes.

\section{Physical fitness}

Physical fitness does seem to be important in helping workers to cope with shift work, so advice on exercise, diet, and sleep management helps the person to manage his or her approach to shift work.

Finally, there is the much debated issue of health surveillance. The Working Time Directive calls for health checks for night workers, although it is unclear what a health check might be! Some suggestions for an effective, and to some extent, an evidence based approach has been proposed by Costa et al. ${ }^{4}$ A sensible and measured stance has also been promulgated in a guidance booklet published jointly by the Society of Occupational Medicine and the Faculty of Occupational Medicine in the United Kingdom. ${ }^{14}$

\section{CONCLUSIONS}

Work involving long hours or abnormal night-day schedules has been in existence for centuries. There is evidence to suggest that such schedules are here to stay, for one reason or another, for one in five workers. The main physiological consequence of such shift schedules is disruption of circadian rhythm which can have a deleterious effect on performance, sleep patterns, accident rates, mental health, and cardiovascular mortality. Reproductive outcome effects may be linked to disruption of menstrual cycles. Individual issues such as sex, age, and personality are also important.

Coping with these potentially harmful work schedules involves helping people to cope and by designing workplace environments and shift scheduling schemes that lead to the least disruption to mental, physical, and social wellbeing.

\section{REFERENCES}

1 Waterhouse JM, Folkard S, Minors DS. Shiftwork, health and safety. An overview of the scientific literature 1978 90. London: The Stationery Office, 1992:1-31

2 Colquhoun WP, Costa G, Folkard S, et al. Shiftwork: problems and solutions.
Frankfurt: Peter Lang, 1996:1-224.

3 Costa G, Folkard S, Harrington JM. Shiftwork and extended hours of work. In: Baxter PJ, Adams PH, Aw TC, et al. Hunters diseases of occupation, 9th ed. London: Arnold, 2000:581-9.

4 Spurgeon A. Working time, occupational health and safety. Geneva: ILO (in press).

- References 1-4 are the best extant reviews of the subject.

5 Folkard S. Circadian performance rhythms: some practical and theoretical implications. Philos Trans R Soc Lond B Biol Sci 1990;327:543-53.

6 Monk TH. Shiftworker performance. Occup Med 1990;5:183-98.

7 Akerstedt T. Psychological and psychophysiological effects of shiftwork. Scand J Work Environ Health 1990:16(suppl 1):67-73.

8 Barton J, Spelton E, Totterdell P, et al. The standard shiftwork index: a battery of questionnaires for assessing shift work-related problems. Work Stress 1995;9:4-30.

9 Harrington JM. Shiftwork and health: a critical review of the literature. London, The Stationery Office, 1978:1-28.

10 Boggild H, Knuttson A. Shift work, risk factors and cardiovascular disease. Scand J Work Environ Health 1999;25:85-99.

- References 5-10 make a good review of the current state of knowledge.

11 Buell P, Breslow L. Mortality from coronary heart disease in Californian men who work long hours. J Chron Dis 1960;11:615-26.

12 Vernon HM. Industrial efficiency and fatigue. In: Collis EL, ed. The industrial clinic. London: John Bale and Sons, 1920:51-74.

13 Spurgeon A, Harrington JM, Cooper CL. Health and safety problems associated with long working hours: a review of the current position. Occup Environ Med 1997;54:367-75.

- Reference 13 is one of the few reviews of long working hours.

14 Society of Occupational Medicine/Faculty of Occupational Medicine. Provision of health assessments under the working time regulations. 1998. A guidance for occupational health physicians and nurses. London: FOM/SOM 1999:1-8.

\section{QUESTIONS (See answers on page 13)}

(1) Shiftwork is needed by industrialised society:
(a) to provide 24 hour emergency service
(b) to offset plant obsolescence
(c) to hasten plant improvements
(d) to improve worker productivity
(e) to maintain continuous process industries

(2) The EC Working Time Directive:

(a) limits hours of work to 48 hours/week averaged over 7 weeks

(b) limits hours of work to 48 hours/week averaged over 17 weeks

(c) limits night work to 24 hours in any 8 days

(d) limits night work to 8 hours in any 24 hours

(e) provides for free health assessments for all shift workers

(3) Circadian rhythms are:
(a) unaltered by external factors
(b) have a 24 hour rhythmicity
(c) lead to peak levels in the late afternoon
(d) can be flattened by shift work
(e) are commonly reversed by shift work

(4) The health effects of shift work include:

(a) decreased quantity but not quality of sleep

(b) increased risk of myocardial infarction

(c) increased risk of dyspepsia

(d) increased risk of spontaneous abortion

(e) increased risk of depressive illness

(5) Shift work is tolerated best by:

(a) the older worker

(b) females

(c) introverts

(d) evening types on night shift

(e) morning types on night shift 